\title{
Radiosynthesis of carbon-11 labeled PDE5 inhibitors as new potential PET radiotracers for imaging of Alzheimer's disease
}

Fugui Dong ${ }^{\mathrm{a}}$, Jie $\mathrm{Du}^{\mathrm{a}}$, Caihong Miao ${ }^{\mathrm{a}}$, Limeng Jia ${ }^{\mathrm{a}}$, Wei Li ${ }^{\mathrm{a}}$, Min Wang ${ }^{\mathrm{b}}$, Qi-Huang Zheng ${ }^{\mathrm{b}, *}$, Zhidong $\mathrm{Xu}^{\mathrm{a}, \mathrm{c}, \mathrm{d}, *}$

${ }^{a}$ Key Laboratory of Medicinal Chemistry and Molecular Diagnosis of Ministry of Education, College of Chemistry and Environmental Science, Hebei University, Baoding, Hebei 071002,

\section{China}

${ }^{b}$ Department of Radiology and Imaging Sciences, Indiana University School of Medicine, 1345 West $16^{\text {th }}$ Street, Room 202, Indianapolis, IN 46202, USA

${ }^{c}$ College of Chemical \& Pharmaceutical Engineering, Key Laboratory of Molecular Chemistry for Medicine of Hebei Province, Hebei University of Science \& Technology, Shijiazhuang, Hebei 050018, China

${ }^{d}$ Shijiazhuang Vince Pharmatech Co., Ltd., Shijiazhuang, Hebei 050030, China

\section{*Corresponding authors:}

Qi-Huang Zheng, Ph.D.

Department of Radiology and Imaging Sciences

Indiana University School of Medicine

1345 West $16^{\text {th }}$ Street, L3-202

This is the author's manuscript of the article published in final edited form as:

Dong, F., Du, J., Miao, C., Jia, L., Li, W., Wang, M., ... Xu, Z. (2019). Radiosynthesis of carbon-11 labeled PDE5 inhibitors as new potential PET radiotracers for imaging of Alzheimer's disease. Applied Radiation and Isotopes, 154, 108873. https://doi.org/10.1016/j.apradiso.2019.108873 
Indianapolis, IN 46202

USA

E-mail: qzheng@iupui.edu

Zhidong Xu, Ph.D.

Key Laboratory of Medicinal Chemistry and Molecular Diagnosis of Ministry of Education College of Chemistry and Environmental Science

Hebei University, Baoding, Hebei 071002

China

E-mail: zhidongxu@hbu.edu.cn 


\begin{abstract}
To develop PET tracers for imaging of Alzheimer's disease, new carbon-11 labeled potent and selective PDE5 inhibitors have been synthesized. The reference standards (5) and (12), and their corresponding desmethylated precursors (6) and (13) were synthesized from methyl 2-amino-5bromobenzoate and (4-methoxyphenyl)methanamine in multiple steps with $2 \%, 1 \%, 1 \%$ and $0.2 \%$ overall chemical yield, respectively. The radiotracers $\left(\left[{ }^{11} \mathrm{C}\right] 5\right)$ and $\left(\left[{ }^{11} \mathrm{C}\right] 12\right)$ were prepared from their corresponding precursors 6 and 13 with $\left[{ }^{11} \mathrm{C}\right] \mathrm{CH}_{3} \mathrm{OTf}$ through $O{ }_{-}{ }^{11} \mathrm{C}$-methylation and isolated by HPLC combined with SPE in $40-50 \%$ radiochemical yield, based on $\left[{ }^{11} \mathrm{C}\right] \mathrm{CO}_{2}$ and decay corrected to EOB. The radiochemical purity was $>99 \%$, and the molar activity $\left(\mathrm{A}_{\mathrm{m}}\right)$ at EOB was in a range of $370-740 \mathrm{GBq} / \mu \mathrm{mol}$.
\end{abstract}

Keywords: Phosphodiesterase 5 (PDE5); Carbon-11 labeled PDE5 inhibitors; Radiosynthesis; Positron emission tomography (PET); Alzheimer's disease (AD).

\title{
1. Introduction
}

Phosphodiesterases (PDEs) are a superfamily of enzymes, consisting of 11 families, that inactivate the second messenger molecules cyclic guanosine monophosphate (cGMP) and cyclic adenosine monophosphate (cAMP), which regulate the extracellular signal transduction and affect fundamental intracellular processes (Andrés et al., 2012; Fiorito et al., 2017; Schröder et al., 2016). Therefore, PDEs modulate various biological processes in both central nervous system (CNS) and peripheral tissues, and are associated with neurological, oncological and 
cardiovascular diseases (Das et al., 2015). PDE has become an attractive therapeutic target, and many PDE inhibitors have been developed for the treatment of PDE-related diseases. In recent years, there has been tremendous interest in identifying new clinical uses of PDE inhibitors in Alzheimer's disease $(\mathrm{AD})$ due to the lack of effective treatments for AD (García-Osta et al., 2012). The continued failure to find effective therapies for $A D$ based on amyloid (A $\beta$ ) hypothesis and tau hypothesis has led researchers to search for new AD management/treatment strategies, and PDEs are promising non-A $\beta$ related candidate targets (Sallustio et al., 2016). Indeed, both PDE4 and PDE5 inhibitors have been found to effectively restore memory function in animal models of AD (García-Osta et al., 2012; Cumming, 2016). Consequently PDEs have become interesting imaging targets in $\mathrm{AD}$, as the development of imaging agents parallels the drug development process (Agdeppa and Spilker, 2009). Advanced biomedical imaging technique positron emission tomography (PET) is a promising modality for $\mathrm{AD}$, and significant advances have occurred in this field of molecular imaging (Frisoni et al., 2017; Johnson et al., 2012). There is a growing interest in design and evaluation of new PET radiotracers for in vivo imaging of PDEs including PDE2, PDE4, PDE5, PDE7 and PDE10 (Andrés et al., 2012; Schröder et al., 2016). In this project of new tracer development, we focus on PDE5 as target, because there are strong preclinical evidences suggesting that PDE5 may serve as a clinically relevant biomarker and a disease-relevant drug/imaging agent target in $\mathrm{AD}$ (Liu et al., 2016; Wenzel et al., 2019). Several PDE5 radioligands have been developed, and representative carbon-11 and fluorine-18 labeled PDE5 inhibitors with an $\mathrm{IC}_{50}$ value $<1 \mathrm{nM}$ are shown in Figure 1, however, so far no successful detection of PDE5 in the brain for quantification of its expression or occupancy has been reported (Chekol et al., 2014; Schröder et al., 2016). This might result from the not high enough inhibitory activity and selectivity of PDE5 radioligands. 
The PDE5 expression in the brain is low with only nanomolar density, and thus, a radioligand with at least subnanomolar PDE5 potency is needed for quantification of this enzyme in brain, but the available PDE5 radioligands only have nanomolar grade IC $_{50}$ values (Chekol et al., 2014; Cumming, 2016; Schröder et al., 2016). Obviously an ideal PDE5 radioligand that can be used in the clinical setting to study PDE5 expression levels in AD remains to be discovered. Recently a novel series of naphthyridine and $1 H$-pyrroloquinolinone analogs have been developed as potent PDE5 inhibitors with picomolar potency for potential treatment of AD, and the excellent lead compounds, 2-acetyl-10-((3-chloro-4-methoxybenzyl)amino)-1,2,3,4tetrahydrobenzo[b][1,6]naphthyridine-8-carbonitrile (5) and 9-((3-chloro-4methoxybenzyl)amino)-2-ethyl-1-oxo-2,3-dihydro- $1 H$-pyrrolo[3,4-b]quinoline-7-carbonitrile (12), exhibited extremely high potency and excellent selectivity with $\mathrm{IC}_{50} 0.056 \mathrm{nM}$ (PDE5A1), $30.1 \mathrm{nM}$ (PDE6C), selectivity (PDE6C/PDE5A1) 537; and 0.059 nM (PDE5A1), $6.6 \mathrm{nM}$ (PDE6C), selectivity (PDE6C/PDE5A1) 112; respectively (Fiorito et al., 2017). These two compounds have the combination of favorable in vitro activity to PDE5, and $O$-methyl positions amenable to labeling with carbon-11, therefore, their carbon-11 labeled radioligands are expected to have high specific binding. Here, we report the design, synthesis and labeling of carbon-11 labeled PDE5 inhibitors, 2-acetyl-10-((3-chloro-4-[ $\left.{ }^{11} \mathrm{C}\right]$ methoxybenzyl)amino)1,2,3,4-tetrahydrobenzo[b][1,6]naphthyridine-8-carbonitrile $\left(\left[{ }^{11} \mathrm{C}\right] 5\right)$ and 9-((3-chloro-4$\left[{ }^{11} \mathrm{C}\right]$ methoxybenzyl)amino)-2-ethyl-1-oxo-2,3-dihydro-1 $H$-pyrrolo[3,4-b]quinoline-7carbonitrile $\left(\left[{ }^{11} \mathrm{C}\right] \mathbf{1 2}\right)$ (Figure 1), as new potential PET radiotracers for imaging of AD.

Insert Figure 1 about here 


\section{Results and discussion}

\subsection{Chemistry}

Synthesis of the reference standard 5 and its desmethylated precursor 2-acetyl-10-((3-chloro-4hydroxybenzyl)amino)-1,2,3,4-tetrahydrobenzo[ $b][1,6]$ naphthyridine-8-carbonitrile (6) is indicated in Scheme 1. Compound $\mathbf{5}$ was synthesized in multiple steps according to the reported procedures (Fiorito et al., 2017; Landry et al., 2015; Schroeder et al., 2014; Snieckus et al., 1972; Yu et al., 2003). The desmethylation of compound 5 with $\mathrm{BBr}_{3}$ in $\mathrm{CH}_{2} \mathrm{Cl}_{2}$ (Aiello et al., 2012; Waghmode et al., 2013) generated the precursor 6 in 30\% yield, which is a new compound.

Insert Scheme 1 about here

Synthesis of the reference standard 12 and its desmethylated precursor 9-((3-chloro-4hydroxybenzyl)amino)-2-ethyl-1-oxo-2,3-dihydro-1H-pyrrolo[3,4- $b]$ quinoline-7-carbonitrile (13) is depicted in Scheme 2. Likewise, compound 12 was synthesized in multiple steps according to the reported procedures (Fiorito et al., 2017; Landry et al., 2015). Similarly, the desmethylation of compound 12 with $\mathrm{BBr}_{3}$ in $\mathrm{CH}_{2} \mathrm{Cl}_{2}$ (Aiello et al., 2012; Waghmode et al., 2013) gave a new compound $\mathbf{1 3}$ in 20\% yield.

Insert Scheme 2 about here 


\subsection{Radiochemistry}

Synthesis of the radiotracers $\left[{ }^{11} \mathrm{C}\right] \mathbf{5}$ and $\left[{ }^{11} \mathrm{C}\right] \mathbf{1 2}$ is shown in Scheme 3. Desmethylated precursor $\mathbf{6}$ or 13 underwent $O-{ }^{11} \mathrm{C}$-methylation (Gao et al., 2018; Wang et al., 2018) using the reactive ${ }^{11} \mathrm{C}$-methylating agent $\left[{ }^{11} \mathrm{C}\right]$ methyl triflate $\left(\left[{ }^{11} \mathrm{C}\right] \mathrm{CH}_{3} \mathrm{OTf}\right)($ Jewett, 1992; Mock et al., 1999) in acetonitrile at $80^{\circ} \mathrm{C}$ under basic conditions $(2 \mathrm{~N} \mathrm{NaOH})$. The product was isolated by semipreparative reversed-phase (RP) high performance liquid chromatography (HPLC) with a C-18 column, and then concentrated by solid-phase extraction (SPE) (Wang et al., 2011, 2012a) with a disposable C-18 Light Sep-Pak cartridge to produce the corresponding pure radiolabeled compound $\left[{ }^{11} \mathrm{C}\right] \mathbf{5}$ or $\left[{ }^{11} \mathrm{C}\right] \mathbf{1 2}$ in $40-50 \%$ radiochemical yield, decay corrected to end of bombardment (EOB), based on $\left[{ }^{11} \mathrm{C}\right] \mathrm{CO}_{2}$.

Insert Scheme 3 about here

The radiosynthesis was performed in a home-built automated multi-purpose ${ }^{11} \mathrm{C}$-radiosynthesis module (Mock et al., 2005a,b; Wang et al., 2012b). Our radiosynthesis module facilitated the overall design of the reaction, purification and reformulation capabilities in a fashion suitable for adaptation to preparation of human doses. The radiosynthesis includes three stages: 1) labeling reaction; 2) purification; and 3) formulation. The overall synthesis time was 35-40 min from EOB. Our module is also designed to allow in-process measurement of ${ }^{11} \mathrm{C}$-tracer molar activity $\left(\mathrm{A}_{\mathrm{m}}, \mathrm{GBq} / \mu \mathrm{mol}\right.$ at $\left.\mathrm{EOB}\right)$ using semi-preparative RP-HPLC (Mock et al., 2005a). At the end of synthesis (EOS), the $\mathrm{A}_{\mathrm{m}}$ of ${ }^{11} \mathrm{C}$-tracer was determined again by analytical RP-HPLC, calculated, decay corrected to EOB, and based on $\left[{ }^{11} \mathrm{C}\right] \mathrm{CO}_{2}$. Both semi-preparative and analytical RP-HPLC 
methods gave similar $A_{m}$ values. The $A_{m}$ of $\left[{ }^{11} \mathrm{C}\right] 5$ and $\left[{ }^{11} \mathrm{C}\right] 12$ at $\mathrm{EOB}$ was in a range of 370-740 $\mathrm{GBq} / \mu \mathrm{mol}$. The general method to increase the $\mathrm{A}_{\mathrm{m}}$ of ${ }^{11} \mathrm{C}$-tracer produced in our radiochemistry facility has been detailed in our previous work (Gao et al., 2018).

Chemical purity and radiochemical purity were determined by analytical HPLC (Zheng and Mock, 2005). A representative analytical RP-HPLC chromatographic profile for the radiotracers $\left[{ }^{11} \mathrm{C}\right] \mathbf{5}$ and $\left[{ }^{11} \mathrm{C}\right] \mathbf{1 2}$, Radio-HPLC (A) and UV-HPLC (B) traces for $\left[{ }^{11} \mathrm{C}\right] \mathbf{5}$; Radio-HPLC (C) and UV-HPLC (D) traces for $\left[{ }^{11} \mathrm{C}\right] \mathbf{1 2}$, is shown in Figure 2. The radiochemical purity of $\left[{ }^{11} \mathrm{C}\right] \mathbf{5}$ or $\left[{ }^{11} \mathrm{C}\right] \mathbf{1 2}$ was $>99 \%$ determined by radio-HPLC through $\gamma$-ray (PIN diode) flow detector as indicated in Figure 2, $\mathbf{A}$ or $\mathbf{C}$. The chemical purity of $\left[{ }^{11} \mathrm{C}\right] \mathbf{5}$ or $\left[{ }^{11} \mathrm{C}\right] \mathbf{1 2}$ was simultaneously determined by UV-HPLC through UV flow detector as indicated in Figure 2, B or D. The minor impurities included its corresponding labeling precursor $\mathbf{6}$ or $\mathbf{1 3}$ and a few unknown UV peaks from the saline used in tracer formulation after HPLC-SPE purification. However, there is no chemical purity of the radiotracer release limit in PET tracer production, because the radiosynthesis is a micro-scale synthesis, and the radiotracer prepared is very trace amount. For non-UV active volatile organic impurities were analyzed and determined by a gas chromatography (GC) equipped with a capillary column and flame ionization detector (FID), and the results met all of the established quality control (QC) criteria.

Insert Figure 2 about here

\section{Experimental}




\subsection{General}

All commercial reagents and solvents were purchased from Sigma-Aldrich and Fisher Scientific, and used without further purification. $\left[{ }^{11} \mathrm{C}\right] \mathrm{CH}_{3} \mathrm{OTf}$ was prepared according to a literature procedure (Mock et al., 1999). Melting points were determined on WRR apparatus and were uncorrected. ${ }^{1} \mathrm{H}$ and ${ }^{13} \mathrm{C}$ NMR spectra were recorded on a Bruker Avance II 400 or $600 \mathrm{MHz}$ NMR Fourier transform spectrometer at 400, 600 or $100 \mathrm{MHz}$, respectively. Chemical shifts $(\delta)$ are reported in parts per million ( $\mathrm{ppm}$ ) relative to an internal standard tetramethylsilane (TMS, $\delta$ $0.0)\left({ }^{1} \mathrm{H}\right.$ NMR $)$ and to the solvent signal $\left({ }^{13} \mathrm{C}\right.$ NMR $)$, and coupling constants $(J)$ are reported in hertz (Hz). Liquid chromatography-mass spectra (LC-MS) analysis was performed on AB Sciex 4000Q Trap instrument, consisting of an 1100 series HPLC connected to a diode array detector and a 1946D mass spectrometer configured for positive-ion/negative-ion electrospray ionization (ESI). The high resolution mass spectra (HRMS) were obtained using a Waters/Micromass LCT Classic spectrometer. Chromatographic solvent proportions are indicated as volume : volume ratio. Thin-layer chromatography (TLC) was run using HS silica gel GF254 uniplates $(5 \times 10$ $\mathrm{cm}^{2}$ ). Plates were visualized under UV light. Normal phase flash column chromatography was carried out on Combiflash Rf 150 silica gel 60 (300-400 mesh) with a forced flow of the indicated solvent system in the proportions described below. All moisture- and air-sensitive reactions were performed under a positive pressure of nitrogen maintained by a direct line from a nitrogen source. Analytical RP HPLC was performed using a Prodigy (Phenomenex) $5 \mu \mathrm{m}$ C-18 column, $4.6 \times 250 \mathrm{~mm}$, mobile phase $65 \% \mathrm{CH}_{3} \mathrm{CN} / 35 \% 4.0 \mathrm{mM} \mathrm{CH}_{3} \mathrm{COONa}$, flow rate 1.0 $\mathrm{mL} / \mathrm{min}$; UV (254 nm) and $\gamma$-ray (PIN diode) flow detectors. Semi-preparative RP HPLC was 
performed using a Prodigy (Phenomenex) $5 \mu \mathrm{m} \mathrm{C}-18$ column, $10 \times 250 \mathrm{~mm} ; 70 \% \mathrm{CH}_{3} \mathrm{CN}: 30 \%$ $\mathrm{H}_{2} \mathrm{O}$ mobile phase; 4 and $5 \mathrm{~mL} / \mathrm{min}$ flow rate for $\left[{ }^{11} \mathrm{C}\right] \mathbf{5}$ and $\left[{ }^{11} \mathrm{C}\right] \mathbf{1 2}$, respectively; $\mathrm{UV}(254 \mathrm{~nm})$ and $\gamma$-ray (PIN diode) flow detectors. C18 Light Sep-Pak cartridges were obtained from Waters Corporation (Milford, MA). Sterile Millex-FG $0.2 \mu \mathrm{m}$ filter units were obtained from Millipore Corporation (Bedford, MA).

\section{2. (3-Chloro-4-methoxyphenyl)methanamine (1)}

A white solid, mp 201.2-201.5 ${ }^{\circ} \mathrm{C} .{ }^{1} \mathrm{H}$ NMR $\left(600 \mathrm{MHz}, \mathrm{CD}_{3} \mathrm{OD}\right): \delta 7.54(\mathrm{~d}, J=2.4 \mathrm{~Hz}, 1 \mathrm{H})$, $7.42(\mathrm{dd}, J=8.4,2.4 \mathrm{~Hz}, 1 \mathrm{H}), 7.15(\mathrm{~d}, J=8.4 \mathrm{~Hz}, 1 \mathrm{H}), 4.08$ (s, 2H), 3.92 (s, 3H). LC-MS (ESI, $m / z)$ : Calcd for $\mathrm{C}_{8} \mathrm{H}_{11} \mathrm{ClNO}\left([\mathrm{M}+\mathrm{H}]^{+}\right)$172.05, found: 172.06 .

\subsection{Methyl 2-amino-5-cyanobenzoate (2)}

A pale yellow solid, mp 131.1-131.9 ${ }^{\circ} \mathrm{C} .{ }^{1} \mathrm{H}$ NMR $\left(600 \mathrm{MHz}, \mathrm{CDCl}_{3}\right): \delta 8.19(\mathrm{~d}, J=1.8 \mathrm{~Hz}$, 1H), 7.45 (dd, $J=9.0,1.8 \mathrm{~Hz}, 1 \mathrm{H}), 6.67(\mathrm{~d}, J=9.0 \mathrm{~Hz}, 1 \mathrm{H}), 6.30$ (br s, 2H), 3.90 (s, 3H). LCMS (ESI, $m / z)$ : Calcd for $\mathrm{C}_{9} \mathrm{H}_{9} \mathrm{~N}_{2} \mathrm{O}_{2}\left([\mathrm{M}+\mathrm{H}]^{+}\right)$177.06, found: 177.00 .

\subsection{2-Amino-5-cyanobenzoic acid (3)}

An off-white solid, mp 265.3-266.5 ${ }^{\circ} \mathrm{C} .{ }^{1} \mathrm{H}$ NMR $\left(600 \mathrm{MHz}, \mathrm{CD}_{3} \mathrm{OD}\right): \delta 8.14(\mathrm{~d}, J=2.4 \mathrm{~Hz}$, 1H), $7.46(\mathrm{dd}, J=9.0,2.4 \mathrm{~Hz}, 1 \mathrm{H}), 6.82(\mathrm{~d}, J=9.0 \mathrm{~Hz}, 1 \mathrm{H}), 4.88$ (br s, 2H). LC-MS (ESI, $m / z)$ : Calcd for $\mathrm{C}_{8} \mathrm{H}_{7} \mathrm{~N}_{2} \mathrm{O}_{2}\left([\mathrm{M}+\mathrm{H}]^{+}\right)$163.05, found: 163.10 . 
3.5. 2-Acetyl-10-chloro-1,2,3,4-tetrahydrobenzo[b][1,6]naphthyridine-8-carbonitrile (4)

A yellow solid, mp 180.2-181.5 ${ }^{\circ} \mathrm{C} .{ }^{1} \mathrm{H}$ NMR (400 MHz, $\mathrm{CDCl}_{3}$ ) (mixture of rotamers): $\delta 8.63$ (d, $J=1.6 \mathrm{~Hz}, 1 \mathrm{H}), 8.11-8.16(\mathrm{~m}, 1 \mathrm{H}), 7.88-7.93(\mathrm{~m}, 1 \mathrm{H}), 5.05$ and $4.93(2 \mathrm{~s}, 2 \mathrm{H}), 4.06$ and 3.94 ( $2 \mathrm{t}, J=6.0 \mathrm{~Hz}, 2 \mathrm{H}), 3.33$ and $3.27(2 \mathrm{t}, J=6.0 \mathrm{~Hz}, 2 \mathrm{H}), 2.32$ and $2.30(2 \mathrm{~s}, 3 \mathrm{H})$. HRMS (ESI, $m / z)$ : Calcd for $\mathrm{C}_{15} \mathrm{H}_{13} \mathrm{ClN}_{3} \mathrm{O}\left([\mathrm{M}+\mathrm{H}]^{+}\right)$286.0742, found: 286.0739 .

3.6. 2-Acetyl-10-((3-chloro-4-methoxybenzyl)amino)-1,2,3,4-tetrahydrobenzo[b] [1,6] naphthyridine-8-carbonitrile (5)

Light yellow oil. ${ }^{1} \mathrm{H}$ NMR (400 MHz, $\mathrm{CDCl}_{3}$ ) (mixture of rotamers): $\delta 8.42-8.47(\mathrm{~m}, 1 \mathrm{H}), 7.96-$ $8.02(\mathrm{~m}, 1 \mathrm{H}), 7.73-7.78(\mathrm{~m}, 1 \mathrm{H}), 7.35-7.42(\mathrm{~m}, 1 \mathrm{H}), 7.17-7.24(\mathrm{~m}, 1 \mathrm{H}), 6.94(\mathrm{~d}, J=8.4 \mathrm{~Hz}, 1 \mathrm{H})$, $4.98(\mathrm{~m}, 1 \mathrm{H}), 4.77(\mathrm{~s}, 2 \mathrm{H}), 4.68(\mathrm{~d}, J=5.6 \mathrm{~Hz}, 2 \mathrm{H}), 3.92(\mathrm{~s}, 3 \mathrm{H}), 3.84(\mathrm{t}, J=6.0 \mathrm{~Hz}, 2 \mathrm{H}), 3.23$ and $3.17(2 \mathrm{t}, J=6.0 \mathrm{~Hz}, 2 \mathrm{H}), 2.23(\mathrm{~s}, 3 \mathrm{H})$. HRMS (ESI, $m / z): \mathrm{C}_{23} \mathrm{H}_{22} \mathrm{ClN}_{4} \mathrm{O}_{2}\left([\mathrm{M}+\mathrm{H}]^{+}\right)$ 421.1426, found: 421.1432 .

3.7. 2-Acetyl-10-((3-chloro-4-hydroxybenzyl)amino)-1,2,3,4-tetrahydrobenzo[b] [1,6] naphthyridine-8-carbonitrile (6)

To a stirred solution of compound $5(50 \mathrm{mg}, 0.12 \mathrm{mmol})$ in $\mathrm{CH}_{2} \mathrm{Cl}_{2}(3 \mathrm{~mL}), \mathrm{BBr}_{3}(60 \mu \mathrm{L}, 0.6$ mmol) was added slowly at $0{ }^{\circ} \mathrm{C}$, and the reaction was continued at $0{ }^{\circ} \mathrm{C}$ for $12 \mathrm{~h}$. The reaction mixture was poured into ice water $(10 \mathrm{~mL})$, and then $\mathrm{CH}_{2} \mathrm{Cl}_{2}$ was removed under reduced 
pressure. The resulted aqueous solution was extracted with EtOAc $(3 \times 20 \mathrm{~mL})$, and the combined organic layer was washed with water, brine, dried over anhydrous $\mathrm{Na}_{2} \mathrm{SO}_{4}$ and filtered. The organic solution was evaporated under vacuum, and the resulted crude product was purified by silica gel column chromatography with $\mathrm{CH}_{2} \mathrm{Cl}_{2} / \mathrm{MeOH}(100: 1$ to $10: 1)$ as eluent to afford $\mathbf{6}$ as a light yellow solid (15 mg, 30\%), mp 114.1-115.8 ${ }^{\circ} \mathrm{C} .{ }^{1} \mathrm{H}$ NMR (400 MHz, $\mathrm{CD}_{3} \mathrm{OD}$ ) (mixture of rotamers): $\delta 8.66(\mathrm{~d}, J=16.0 \mathrm{~Hz}, 1 \mathrm{H}), 7.90-7.95(\mathrm{~m}, 1 \mathrm{H}), 7.81-7.86(\mathrm{~m}, 1 \mathrm{H}), 7.33-7.40(\mathrm{~m}, 1 \mathrm{H})$, 7.13-7.17 (m, 1H), 6.88-6.95 (m, 1H), 4.85 and $4.75(2 \mathrm{~s}, 2 \mathrm{H}), 4.74(\mathrm{~s}, 2 \mathrm{H}), 3.90-3.93(\mathrm{~m}, 2 \mathrm{H})$, 3.21 and $3.11(2 \mathrm{t}, J=6.4 \mathrm{~Hz}, 2 \mathrm{H}), 2.25(\mathrm{~s}, 2 \mathrm{H}), 2.00(\mathrm{~d}, J=8.0 \mathrm{~Hz}, 3 \mathrm{H}) .{ }^{13} \mathrm{C}$ NMR $(100 \mathrm{MHz}$, $\mathrm{CD}_{3} \mathrm{OD}$ ) (mixture of rotamers): $\delta 169.14,168.89,159.88,152.48,149.44,149.34,148.91$, $148.83,132.23,131.95,130.25,129.71,129.12,128.88,127.26,127.04,120.13,119.98,119.76$, $117.12,117.02,113.88,113.16,106.30,106.22,50.10,45.08,43.27,33.77,33.14,21.84,21.76$. HRMS (ESI, $m / z): \mathrm{C}_{22} \mathrm{H}_{20} \mathrm{ClN}_{4} \mathrm{O}_{2}\left([\mathrm{M}+\mathrm{H}]^{+}\right)$407.1269, found: 407.1273.

\subsection{2,4-Dioxo-1,4-dihydro-2H-benzo[d][1,3]oxazine-6-carbonitrile (7)}

A light yellow solid, mp 269.6-271.3 ${ }^{\circ} \mathrm{C} .{ }^{1} \mathrm{H}$ NMR (400 MHz, CD $\left.{ }_{3} \mathrm{OD}\right): \delta 8.36$ (d, $J=2.0 \mathrm{~Hz}$, 1H), $8.00(\mathrm{dd}, J=8.4,2.0 \mathrm{~Hz}, 1 \mathrm{H}), 7.26(\mathrm{~d}, J=8.4 \mathrm{~Hz}, 1 \mathrm{H})$. LC-MS (ESI, $m / z)$ : Calcd for $\mathrm{C}_{9} \mathrm{H}_{5} \mathrm{~N}_{2} \mathrm{O}_{3}\left([\mathrm{M}+\mathrm{H}]^{+}\right)$189.02, found: 188.10 .

3.9. Methyl 6-cyano-2-methyl-4-oxo-1,4-dihydroquinoline-3-carboxylate (8) 
A yellow solid, mp $300{ }^{\circ} \mathrm{C}$ (Dec.). ${ }^{1} \mathrm{H}$ NMR (400 MHz, DMSO-d6): $\delta 12.31$ (br s, $\left.1 \mathrm{H}\right), 8.42$ (s, 1H), $8.04(\mathrm{~d}, J=8.8 \mathrm{~Hz}, 1 \mathrm{H}), 7.69(\mathrm{~d}, J=8.8 \mathrm{~Hz}, 1 \mathrm{H}), 3.81$ (s, 3H), 2.44 (s, 3H). LC-MS (ESI, $m / z)$ : Calcd for $\mathrm{C}_{13} \mathrm{H}_{11} \mathrm{~N}_{2} \mathrm{O}_{3}\left([\mathrm{M}+\mathrm{H}]^{+}\right)$243.07, found: 243.10 .

\subsection{Methyl 4-chloro-6-cyano-2-methylquinoline-3-carboxylate (9)}

A white solid, mp 157.2-158.3 ${ }^{\circ} \mathrm{C} .{ }^{1} \mathrm{H}$ NMR $\left(400 \mathrm{MHz}, \mathrm{CDCl}_{3}\right): \delta 8.66(\mathrm{~s}, 1 \mathrm{H}), 8.17(\mathrm{~d}, J=8.4$

Hz, 1H), 7.97 (d, $J=8.4 \mathrm{~Hz}, 1 \mathrm{H}), 4.11$ (s, 3H), 2.80 (s, 3H). LC-MS (ESI, $m / z)$ : Calcd for $\mathrm{C}_{13} \mathrm{H}_{10} \mathrm{ClN}_{2} \mathrm{O}_{2}\left([\mathrm{M}+\mathrm{H}]^{+}\right)$261.04, found: 261.00 .

3.11. Methyl 2-(bromomethyl)-4-chloro-6-cyanoquinoline-3-carboxylate (10)

A light yellow solid, mp 108.5-109.9 ${ }^{\circ} \mathrm{C} .{ }^{1} \mathrm{H}$ NMR $\left(600 \mathrm{MHz}, \mathrm{CDCl}_{3}\right): \delta 8.67(\mathrm{~d}, J=1.8 \mathrm{~Hz}$, 1H), $8.19(\mathrm{~d}, J=8.4 \mathrm{~Hz}, 1 \mathrm{H}), 7.98(\mathrm{dd}, J=8.4,1.8 \mathrm{~Hz}, 1 \mathrm{H}), 4.79$ (s, 2H), 4.10 (s, 3H). LC-MS (ESI, $m / z)$ : Calcd for $\mathrm{C}_{13} \mathrm{H}_{9} \mathrm{BrClN}_{2} \mathrm{O}_{2}\left([\mathrm{M}+\mathrm{H}]^{+}\right)$340.94, found: 341.10 .

\subsection{9-Chloro-2-ethyl-1-oxo-2,3-dihydro-1H-pyrrolo[3,4-b]quinoline-7-carbonitrile (11)}

A white solid, mp 195.5-196.1 ${ }^{\circ} \mathrm{C} .{ }^{1} \mathrm{H}$ NMR $\left(600 \mathrm{MHz}, \mathrm{CDCl}_{3}\right): \delta 8.84(\mathrm{~d}, J=1.2 \mathrm{~Hz}, 1 \mathrm{H}), 8.24$ $(\mathrm{d}, J=9.0 \mathrm{~Hz}, 1 \mathrm{H}), 8.00(\mathrm{dd}, J=9.0,1.2 \mathrm{~Hz}, 1 \mathrm{H}), 4.58(\mathrm{~s}, 2 \mathrm{H}), 3.78(\mathrm{q}, J=7.2 \mathrm{~Hz}, 2 \mathrm{H}), 1.35$ (t, $J=7.2 \mathrm{~Hz}, 3 \mathrm{H})$. HRMS (ESI, $m / z)$ : Calcd for $\mathrm{C}_{14} \mathrm{H}_{11} \mathrm{ClN}_{3} \mathrm{O}\left([\mathrm{M}+\mathrm{H}]^{+}\right) 272.0585$, found: 272.0585 . 


\subsection{9-((3-Chloro-4-methoxybenzyl)amino)-2-ethyl-1-oxo-2,3-dihydro-1H-pyrrolo[3,4-}

b]quinoline-7-carbonitrile (12)

A white solid, mp 205.4-206.6 ${ }^{\circ} \mathrm{C} .{ }^{1} \mathrm{H}$ NMR $\left(400 \mathrm{MHz}, \mathrm{CD}_{3} \mathrm{Cl}\right): \delta 8.63(\mathrm{t}, J=5.6 \mathrm{~Hz}, 1 \mathrm{H}), 8.58$

(s, 1H), $8.00(\mathrm{~d}, J=8.8 \mathrm{~Hz}, 1 \mathrm{H}), 7.80(\mathrm{dd}, J=8.8,1.6 \mathrm{~Hz}, 1 \mathrm{H}), 7.48$ (d, $J=1.6 \mathrm{~Hz}, 1 \mathrm{H}), 7.36$

(dd, $J=8.4,1.6 \mathrm{~Hz}, 1 \mathrm{H}), 7.02(\mathrm{~d}, J=8.4 \mathrm{~Hz}, 1 \mathrm{H}), 4.99(\mathrm{~d}, J=5.6 \mathrm{~Hz}, 2 \mathrm{H}), 4.44(\mathrm{~s}, 2 \mathrm{H}), 3.96(\mathrm{~s}$, 3H), 3.69 (q, $J=7.2 \mathrm{~Hz}, 2 \mathrm{H}), 1.33$ (t, $J=7.2 \mathrm{~Hz}, 3 \mathrm{H})$. HRMS (ESI, $m / z)$ : Calcd for $\mathrm{C}_{22} \mathrm{H}_{20} \mathrm{ClN}_{4} \mathrm{O}_{2}\left([\mathrm{M}+\mathrm{H}]^{+}\right)$407.1275, found: 407.1273.

\subsection{9-((3-Chloro-4-hydroxybenzyl)amino)-2-ethyl-1-oxo-2,3-dihydro-1H-pyrrolo[3,4-} b]quinoline-7-carbonitrile (13)

To a stirred solution of compound $12(25 \mathrm{mg}, 0.061 \mathrm{mmol})$ in $\mathrm{CH}_{2} \mathrm{Cl}_{2}(2 \mathrm{~mL}), \mathrm{BBr}_{3}(30 \mu \mathrm{L}, 0.3$ mmol) was added slowly at $0{ }^{\circ} \mathrm{C}$, and the reaction was continued at $0{ }^{\circ} \mathrm{C}$ for $20 \mathrm{~h}$. The reaction mixture was poured into ice water $(5 \mathrm{~mL})$, and then $\mathrm{CH}_{2} \mathrm{Cl}_{2}$ was removed under reduced pressure. The resulted aqueous solution was extracted with EtOAc $(3 \times 10 \mathrm{~mL})$, and the combined organic layer was washed with water, brine, dried over anhydrous $\mathrm{Na}_{2} \mathrm{SO}_{4}$ and filtered. The organic solution was evaporated under vacuum, and the resulted crude product was purified by silica gel column chromatography with $\mathrm{CH}_{2} \mathrm{Cl}_{2} / \mathrm{MeOH}$ (100:1 to 25:1) as eluent to afford $\mathbf{1 3}$ as a light yellow solid (5 mg, 20\%), mp 185.1-186.4 ${ }^{\circ} \mathrm{C} .{ }^{1} \mathrm{H}$ NMR (400 MHz, DMSO-d6): $\delta 9.09$ (br s, 1H), $8.53(\mathrm{~m}, 1 \mathrm{H}) .8 .09(\mathrm{~d}, J=8.4 \mathrm{~Hz}, 1 \mathrm{H}), 8.00(\mathrm{~d}, J=8.4 \mathrm{~Hz}, 1 \mathrm{H}), 7.47(\mathrm{~d}, J=2.0 \mathrm{~Hz}$, $1 \mathrm{H}), 7.26(\mathrm{dd}, J=8.4,2.0 \mathrm{~Hz}, 1 \mathrm{H}), 7.06(\mathrm{~d}, J=8.4 \mathrm{~Hz}, 1 \mathrm{H}), 5.40(\mathrm{~d}, J=4.8 \mathrm{~Hz}, 2 \mathrm{H}), 4.56(\mathrm{~s}$, $2 \mathrm{H}), 3.64(\mathrm{q}, J=7.2 \mathrm{~Hz}, 2 \mathrm{H}), 3.48(\mathrm{br} \mathrm{s}, 1 \mathrm{H}), 1.30(\mathrm{t}, J=7.2 \mathrm{~Hz}, 3 \mathrm{H}) .{ }^{13} \mathrm{C}$ NMR $(150 \mathrm{MHz}$, 
DMSO-d6): $\delta 166.70,165.95,152.30,151.31,149.50,131.34,131.14,130.74,130.00,129.06$, 127.26, 119.44, 118.95, 118.51, 116.67, 106.06, 104.16, 50.23, 48.70, 36.30, 12.98. HRMS (ESI, $m / z)$ : Calcd for $\mathrm{C}_{21} \mathrm{H}_{18} \mathrm{ClN}_{4} \mathrm{O}_{2}\left([\mathrm{M}+\mathrm{H}]^{+}\right)$393.1118, found: 393.1117 .

\subsection{2-Acetyl-10-((3-chloro-4- $\left[{ }^{11} C\right]$ methoxybenzyl)amino)-1,2,3,4-}

tetrahydrobenzo[b] $[1,6]$ naphthyridine-8-carbonitrile $\left(\left[{ }^{11} C\right] 5\right)$ and 9-((3-chloro-4-

$\left[{ }^{11}\right.$ C]methoxybenzyl)amino)-2-ethyl-1-oxo-2,3-dihydro-1H-pyrrolo[3,4-b]quinoline-7carbonitrile $\left(\left[{ }^{11} C\right] 12\right)$

$\left[{ }^{11} \mathrm{C}\right] \mathrm{CO}_{2}$ was produced by the ${ }^{14} \mathrm{~N}(\mathrm{p}, \alpha){ }^{11} \mathrm{C}$ nuclear reaction in the small volume $\left(9.5 \mathrm{~cm}^{3}\right)$ aluminum gas target provided with the Siemens RDS-111 Eclipse cyclotron. The target gas consisted of $1 \%$ oxygen in nitrogen purchased as a specialty gas from Praxair, Indianapolis, IN. Typical irradiations used for the development were $58 \mu \mathrm{A}$ beam current and 15 min on target. The production run produced approximately $25.9 \mathrm{GBq}$ of $\left[{ }^{11} \mathrm{C}^{-} \mathrm{CO}_{2}\right.$ at EOB. Desmethylated precursor 6 or $13(0.1-0.3 \mathrm{mg})$ was dissolved in $\mathrm{CH}_{3} \mathrm{CN}(300 \mu \mathrm{L})$. To this solution was added aqueous $\mathrm{NaOH}(2 \mathrm{~N}, 2 \mu \mathrm{L})$. The mixture was transferred to a small reaction vial. No-carrieradded (high molar activity) $\left[{ }^{11} \mathrm{C}\right] \mathrm{CH}_{3} \mathrm{OTf}$ that was produced by the gas-phase production method (Mock et al., 1999) within 12 min from $\left[{ }^{11} \mathrm{C}^{\mathrm{C}} \mathrm{CO}_{2}\right.$ through $\left[{ }^{11} \mathrm{C}\right] \mathrm{CH}_{4}$ and $\left[{ }^{11} \mathrm{C}\right] \mathrm{CH}_{3} \mathrm{Br}$ with silver triflate (AgOTf) column was passed into the reaction vial at room temperature (RT) until radioactivity reached a maximum $(2 \mathrm{~min})$, and then the reaction vial was isolated and heated at $80{ }^{\circ} \mathrm{C}$ for 3 min. The contents of the reaction vial were diluted with aqueous $\mathrm{NaHCO}_{3}(0.1 \mathrm{M}, 1$ $\mathrm{mL}$ ). The reaction vial was connected to a $3 \mathrm{~mL}$ HPLC injection loop. The labeled product mixture solution was injected onto the semi-preparative HPLC column for purification. The 
product fraction was collected in a recovery vial containing $30 \mathrm{~mL}$ water. The diluted tracer solution was then passed through a C-18 Sep-Pak Light cartridge, and washed with water $(3 \times 10$ $\mathrm{mL})$. The cartridge was eluted with $\mathrm{EtOH}(3 \times 0.4 \mathrm{~mL})$ to release the labeled product, followed by saline $(10-11 \mathrm{~mL})$. The eluted product was then sterile-filtered through a Millex-FG $0.2 \mu \mathrm{m}$ membrane into a sterile vial. Total radioactivity was assayed and total volume $(10-11 \mathrm{~mL})$ was noted for tracer dose dispensing. The overall synthesis time including HPLC-SPE purification and reformulation was 35-40 min from EOB. The decay corrected radiochemical yield was 40$50 \%$. Retention times $\left(t_{\mathrm{R}}\right)$ in the analytical RP-HPLC system were: $t_{\mathrm{R}} \mathbf{6}=3.24 \mathrm{~min}, \mathrm{t}_{\mathrm{R}} \mathbf{5}=4.21$ $\min$, and $t_{R}\left[{ }^{11} \mathrm{C}\right] \mathbf{5}=4.30 \mathrm{~min}$; and $\mathrm{t}_{\mathrm{R}} \mathbf{1 3}=3.33 \mathrm{~min}, \mathrm{t}_{\mathrm{R}} \mathbf{1 2}=5.52 \mathrm{~min}$, and $\mathrm{t}_{\mathrm{R}}\left[{ }^{11} \mathrm{C}\right] \mathbf{1 2}=5.64 \mathrm{~min}$. Retention times in the semi-preparative RP-HPLC system were: $t_{R} \mathbf{6}=5.51 \mathrm{~min}, \mathrm{t}_{\mathrm{R}} \mathbf{5}=7.07 \mathrm{~min}$, and $t_{R}\left[{ }^{11} \mathrm{C}\right] \mathbf{5}=7.12 \mathrm{~min}$; and $\mathrm{t}_{\mathrm{R}} \mathbf{1 3}=3.89 \mathrm{~min}, \mathrm{t}_{\mathrm{R}} \mathbf{1 2}=6.88 \mathrm{~min}$, and $\mathrm{t}_{\mathrm{R}}\left[{ }^{11} \mathrm{C}\right] \mathbf{1 2}=6.93 \mathrm{~min}$.

\section{Conclusion}

In summary, multiple step synthetic routes with reasonable yields have been developed to produce the precursors 6 and 13, the reference standards 5 and 12, and the target PET radiotracers $\left[{ }^{11} \mathrm{C}\right] \mathbf{5}$ and $\left[{ }^{11} \mathrm{C}\right] \mathbf{1 2}$. The radiosynthesis employed $\left[{ }^{11} \mathrm{C}\right] \mathrm{CH}_{3} \mathrm{OTf}$ for $O-{ }^{11} \mathrm{C}$ methylation at the phenyl hydroxyl position of the precursor, followed by product purification and isolation by a semi-preparative RP-HPLC combined with SPE. $\left[{ }^{11} \mathrm{C}\right] \mathbf{5}$ and $\left[{ }^{11} \mathrm{C}\right] \mathbf{1 2}$ were obtained in high radiochemical yield, and high radiochemical purity, with a reasonably short overall synthesis time, and high molar activity. Two new carbon-11 labeled potent and selective 
PDE5 inhibitors have been successfully radiosynthesized. This will facilitate studies to evaluate $\left[{ }^{11} \mathrm{C}\right] \mathbf{5}$ and $\left[{ }^{11} \mathrm{C}\right] \mathbf{1 2}$ as new potential PET agents for imaging of PDE5 in AD.

\section{Conflict of interest statement}

The authors declare that they have no conflict of interest relevant to this article.

\section{Acknowledgments}

This research was partially supported by the Hebei Province Major Science and Technology Program (No. 17392605D), and the High Level Scientific and Technological Innovation and Entrepreneurial Talent Plan of Shijiazhuang, China. This work was also partially supported by the Indiana University Department of Radiology and Imaging Sciences in the United States.

\section{Appendix A. Supplementary material}

Supplementary data associated with this article can be found in the online version at doi:

\section{References}


Agdeppa, E.D., Spilker, M.E., 2009. A review of imaging agent development. AAPS J. 11, 286299.

Aiello, F., Shabaik, Y., Esqueda, A., Sanchez, T.W., Grande, F., Garofalo, A., Neamati, N., 2012. Design and synthesis of 3-carbamoylbenzoic acid derivatives as inhibitors of human apurinic/apyrimidinic endonuclease 1 (APE1). ChemMedChem. 7. 1825-1839.

Andrés, J.I., De Angelis, M., Alcázar, J., Celen, S., Bormans, G., 2012. Recent advances in positron emission tomography (PET) radiotracers for imaging phosphodiesterases. Curr. Top. Med. Chem. 12, 1224-1236.

Chekol, R., Gheysens, O., Cleynhens, J., Pokreisz, P., Vanhoof, G., Ahamed, M., Janssens, S., Verbruggen, A., Bormans, G., 2014. Evaluation of PET radioligands for in vivo visualization of phosphodiesterase 5 (PDE5). Nucl. Med. Biol. 41, 155-162.

Cumming, P., 2016. A business of some heat: molecular imaging of phosphodiesterase 5. J. Neurochem. 136. 220-221.

Das, A., Durrant, D., Salloum, F.N., Xi, L., Kukreja, R.C., 2015. PDE5 inhibitors as therapeutics for heart disease, diabetes and cancer. Pharmacol. Ther. 147, 12-21.

Fiorito, J., Vendome, J., Saeed, F., Staniszewski, A., Zhang, H., Yan, S., Deng, S.-X., Arancio, O., Landry, D.W., 2017. Identification of a novel 1,2,3,4tetrahydrobenzo $[b][1,6]$ naphthyridine analogue as a potent phosphodiesterase 5 inhibitor with improved aqueous solubility for the treatment of Alzheimer's disease. J. Med. Chem. 60, 8858-8875.

Frisoni, G.B., Boccardi, M., Barkhof, F., Blennow, K., Cappa, S., Chiotis, K., Démonet, J.F., Garibotto, V., Giannakopoulos, P., Gietl, A., Hansson, O., Herholz, K., Jack, C.R. Jr., Nobili, 
F., Nordberg, A., Snyder, H.M., Ten Kate, M., Varrone, A., Albanese, E., Becker, S., Bossuyt, P., Carrillo, M.C., Cerami, C., Dubois, B., Gallo, V., Giacobini, E., Gold, G., Hurst, S., Lönneborg, A., Lovblad, K.O., Mattsson, N., Molinuevo, J.L., Monsch, A.U., Mosimann, U., Padovani, A., Picco, A., Porteri, C., Ratib, O., Saint-Aubert, L., Scerri, C., Scheltens, P., Schott, J.M., Sonni, I., Teipel, S., Vineis, P., Visser, P.J., Yasui, Y., Winblad, B., 2017.

Strategic roadmap for an early diagnosis of Alzheimer's disease based on biomarkers. Lancet Neurol. 16, 661-676.

Gao, M., Wang M., Zheng, Q.-H., 2018. Synthesis of carbon-11-labeled CK1 inhibitors as new potential PET radiotracers for imaging of Alzheimer's disease. Bioorg. Med. Chem. Lett. 28, 2234-2238.

García-Osta, A., Cuadrado-Tejedor, M., García-Barroso, C., Oyarzábal, J., Franco, R., 2012. Phosphodiesterases as therapeutic targets for Alzheimer's disease. ACS Chem. Neurosci. 3, 832-844.

Jewett, D.M., 1992. A simple synthesis of $\left[{ }^{11}\right.$ C $]$ methyl triflate. Int. J. Rad. Appl. Instrum. A 43, 1383-1385.

Johnson, K.A., Fox, N.C., Sperling, R.A., Klunk, W.E., 2012. Brain imaging in Alzheimer disease. Cold Spring Harb. Perspect. Med. 2, a006213.

Landry, D.W., Deng, X.-S., Fiorito, J., Arancio, O., Wasmuth, A.A., 2015. Preparation of benzonaphthyridine derivatives as phosphodiesterase inhibitors for treatment of neurodegenerative diseases. WO 2015009930.

Liu, J., Wenzel, B., Dukic-Stefanovic, S., Teodoro, R., Ludwig, F.A., Deuther-Conrad, W., Schröder, S., Chezal, J.M., Moreau, E., Brust, P., Maisonial-Besset, A., 2016. Development 
of a new radiofluorinated quinolone analog for PET imaging of phosphodiesterase 5 (PDE5) in brain. Pharmaceuticals (Basel). 9, E22.

Mock, B.H., Mulholland, G.K., Vavrek, M.T., 1999. Convenient gas phase bromination of $\left[{ }^{11} \mathrm{C}\right]$ methane and production of $\left[{ }^{11} \mathrm{C}\right]$ methyl triflate. Nucl. Med. Biol. 26, 467-471.

Mock, B.H., Glick-Wilson, B.E., Zheng, Q.-H., DeGrado, T.R., 2005a. Automated measurement of specific activity of radiolabeled ligands during synthesis. J. Label. Compd. Radiopharm. $48, \mathrm{~S} 224$.

Mock, B.H., Zheng, Q.-H., DeGrado, T.R., 2005b. A multi-purpose ${ }^{11}$ C-radio-synthesis system. J. Label. Compd. Radiopharm. 48, S225.

Sallustio, F., Studer, V., 2016. Targeting new pharmacological approaches for Alzheimer's disease: Potential for statins and phosphodiesterase inhibitors. CNS Neurol. Disord. Drug Targets. 15, 647-659.

Schröder, S., Wenzel, B., Deuther-Conrad, W., Scheunemann, M., Brust, P., 2016. Novel radioligands for cyclic nucleotide phosphodiesterase imaging with positron emission tomography: An update on developments since 2012. Molecules. 21, E650.

Schroeder, C.E., Yao, T., Sotsky, J., Smith, R.A., Roy, S., Chu, Y.K., Guo, H., Tower, N.A., Noah, J.W., McKellip, S., Sosa, M., Rasmussen, L., Smith, L.H., White, E.L., Aubé, J., Jonsson, C.B., Chung, D., Golden, J.E., 2014. Development of (E)-2-((1,4dimethylpiperazin-2-ylidene)amino)-5-nitro- $N$-phenylbenzamide, ML336: Novel 2amidinophenylbenzamides as potent inhibitors of venezuelan equine encephalitis virus. J. Med. Chem. 57, 8608-8621.

Snieckus, V.A., Onouchi, T., Boekelheide, V., 1972. Stereoselective syntheses of isoquinuclidones. I. J. Org. Chem. 37, 2845-2848. 
Waghmode, S.B., Mahale, G., Patil, V.P., Renalson, K., Singh, D., 2013. Efficient method for demethylation of aryl methyl ether using aliquat-336. Synth. Commun. 43, 3272-3280.

Wang, M., Gao, M., Miller, K.D., Zheng, Q.-H., 2011. Synthesis of $\left[{ }^{11}\right.$ C $]$ PBR06 and $\left[{ }^{18}\right.$ F]PBR06 as agents for positron emission tomographic (PET) imaging of the translocator protein (TSPO). Steroids 76, 1331-1340.

Wang, M., Gao, M., Miller, K.D., Sledge, G.W., Zheng, Q.-H., 2012a. [ ${ }^{11}$ C]GSK2126458 and $\left[{ }^{18} \mathrm{~F}\right] \mathrm{GSK} 2126458$, the first radiosynthesis of new potential PET agents for imaging of PI3K and mTOR in cancers. Bioorg. Med. Chem. Lett. 22, 1569-1574.

Wang, M., Gao, M., Zheng, Q.-H., 2012b. Fully automated synthesis of PET TSPO radioligands $\left[{ }^{11} \mathrm{C}\right] \mathrm{DAA} 1106$ and $\left[{ }^{18} \mathrm{~F}\right] \mathrm{FEDAA} 1106$. Appl. Radiat. Isot. 70, 965-973.

Wang, X., Dong, F., Miao, C., Li, W., Wang, M., Gao, M., Zheng, Q.-H., Xu, Z., 2018. Synthesis of carbon-11-labeled 5- $\mathrm{HT}_{6} \mathrm{R}$ antagonists as new candidate PET radioligands for imaging of Alzheimer's disease. Bioorg. Med. Chem. Lett. 28, 1836-1841.

Wenzel, B., Liu, J., Dukic-Stefanovic, S., Deuther-Conrad, W., Teodoro, R., Ludwig, F.A., Chezal, J.M., Moreau, E., Brust, P., Maisonial-Besset, A., 2019. Targeting cyclic nucleotide phosphodiesterase 5 (PDE5) in brain: Toward the development of a PET radioligand labeled with fluorine-18. Bioorg Chem. 86, 346-362.

Yu, G., Mason, H.J., Galdi, K., Wu, X., Cornelius, L., Zhao, N., Witkus, M., Ewing, W.R., Macor, J.E., 2003. Chlorination of arylalkyl- and heteroarylalkyl amines and amino acids using sulfuryl chloride. Synthesis. 403-407.

Zheng, Q.-H., Mock, B.H., 2005. Purification of carbon-11 PET radiotracers from unlabeled precursors by preparative HPLC and SPE. Biomed. Chromatogr. 19, 671-676. 


\section{Figure and Scheme Legends}

Figure 1. PET PDE5 radioligands.

Figure 2. A representative analytical RP-HPLC chromatographic profile for the radiotracers $\left[{ }^{11} \mathrm{C}\right] \mathbf{5}$ and $\left[{ }^{11} \mathrm{C}\right]$ 12: (A) Radio-HPLC trace for $\left[{ }^{11} \mathrm{C}\right] \mathbf{5}$ and $(\mathbf{B}) \mathrm{UV}-\mathrm{HPLC}$ trace for $\left[{ }^{11} \mathrm{C}\right] \mathbf{5}$; (C) Radio-HPLC trace for $\left[{ }^{11} \mathrm{C}\right] 12$ and (D) UV-HPLC trace for $\left[{ }^{11} \mathrm{C}\right] \mathbf{1 2}$. Analytical RP-HPLC conditions were a Prodigy (Phenomenex) $5 \mu \mathrm{m}$ C-18 column, $4.6 \times 250 \mathrm{~mm}$; mobile phase $65 \%$ $\mathrm{CH}_{3} \mathrm{CN} / 35 \%$ 4.0 $\mathrm{mM} \mathrm{CH} 3 \mathrm{COONa}_{3}$ flow rate $1.0 \mathrm{~mL} / \mathrm{min}$; $\mathrm{UV}(254 \mathrm{~nm})$ and $\gamma$-ray (PIN diode) flow detectors.

Scheme 1. Synthesis of the reference standard (5) and precursor (6). Conditions: (i) $\mathrm{CuCN}, N$ methyl-2-pyrrolidone (NMP), $200{ }^{\circ} \mathrm{C}$; (ii) $\mathrm{KOH}, \mathrm{EtOH}, \mathrm{H}_{2} \mathrm{O}, 60{ }^{\circ} \mathrm{C}$; (iii) 1-acetylpiperidin-4-one, $\mathrm{POCl}_{3}, 60^{\circ} \mathrm{C}$; (iv) 1, $N, N$-diisopropylethylamine (DIPEA), $n$-propanol, reflux; (v) $\mathrm{BBr}_{3}, \mathrm{CH}_{2} \mathrm{Cl}_{2}$, $0{ }^{\circ} \mathrm{C}$

Scheme 2. Synthesis of the reference standard (12) and precursor (13). Conditions: (i) triphosgene, 1,4-dioxane, $90{ }^{\circ} \mathrm{C}$; (ii) $\mathrm{NaH}$, methyl acetoacetate, dimethylamine (DMA), $90{ }^{\circ} \mathrm{C}$; (iii) $\mathrm{POCl}_{3}, 110^{\circ} \mathrm{C}$; (iv) 2,2'-azobis(2-methylpropionitrile), $N$-bromosuccinimide (NBS), $\mathrm{CCl}_{4}$, reflux; (v) EtNH 2 , EtOH, THF, $78^{\circ} \mathrm{C}$; (vi) 1, DIPEA, $n$-propanol, $90^{\circ} \mathrm{C}$, (vii) $\mathrm{BBr}_{3}, \mathrm{CH}_{2} \mathrm{Cl}_{2}, 0$ ${ }^{\circ} \mathrm{C}$.

Scheme 3. Synthesis of the radiotracers $\left(\left[{ }^{11} \mathrm{C}\right] \mathbf{5}\right)$ and $\left(\left[{ }^{11} \mathrm{C}\right] \mathbf{1 2}\right)$. 
Figure 1.

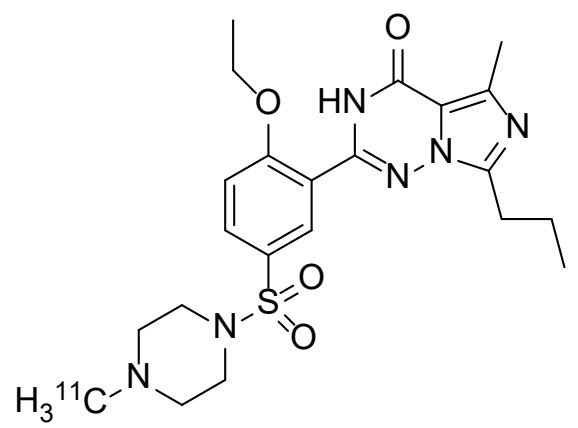

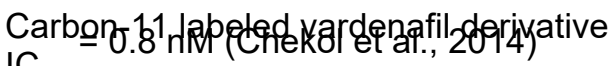
$\mathrm{IC}_{50}$

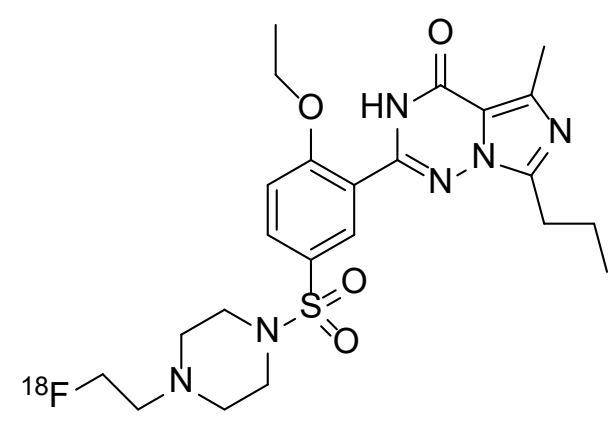

Fluorine-7 8 . $\mathrm{IC}_{50}$<smiles>CCOc1ccc(OCCN2C(=O)c3c(nc4ccc(C#N)cc4c3NCc3ccc(OC)c(Cl)c3)CN2CC)cc1</smiles> 


\section{Figure 2.}

A. Analytical radioactive HPLC trace for $\left[{ }^{11} \mathrm{C}\right] 5$, Retention time $\left(t_{R}\right)=4.30 \mathrm{~min}$

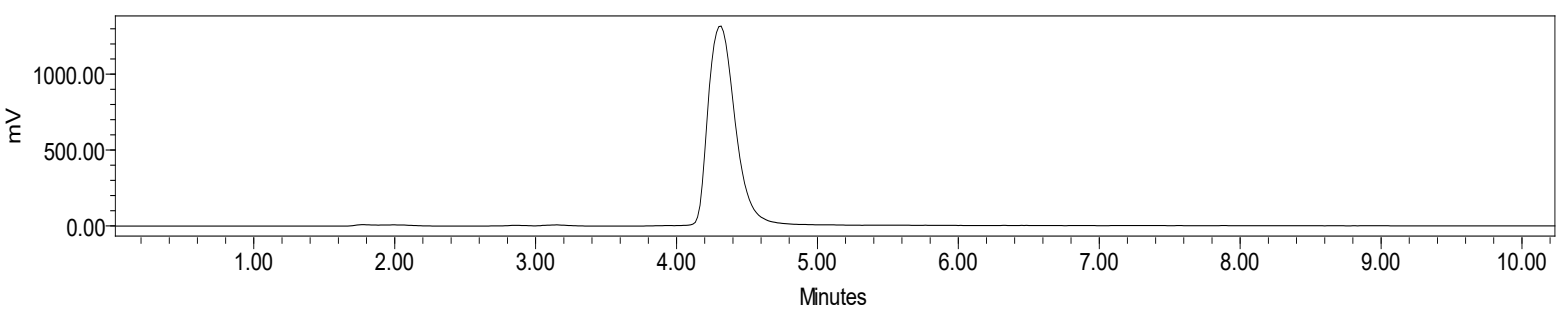

B. Analytical UV HPLC trace for $\left[{ }^{11} \mathrm{C}\right] \mathbf{5}, \mathrm{t}_{\mathrm{R}}=4.21 \mathrm{~min}$

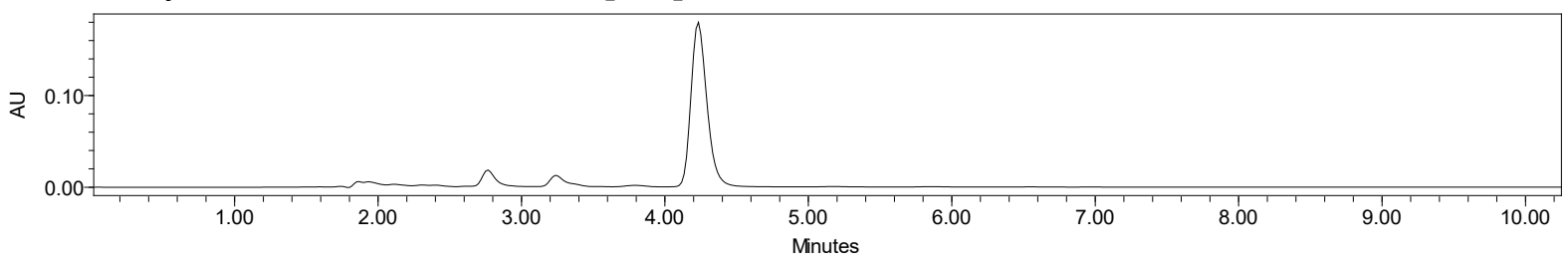

C. Analytical radioactive HPLC trace for $\left[{ }^{11} \mathrm{C}\right] 12, \mathrm{t}_{\mathrm{R}}=5.64 \mathrm{~min}$

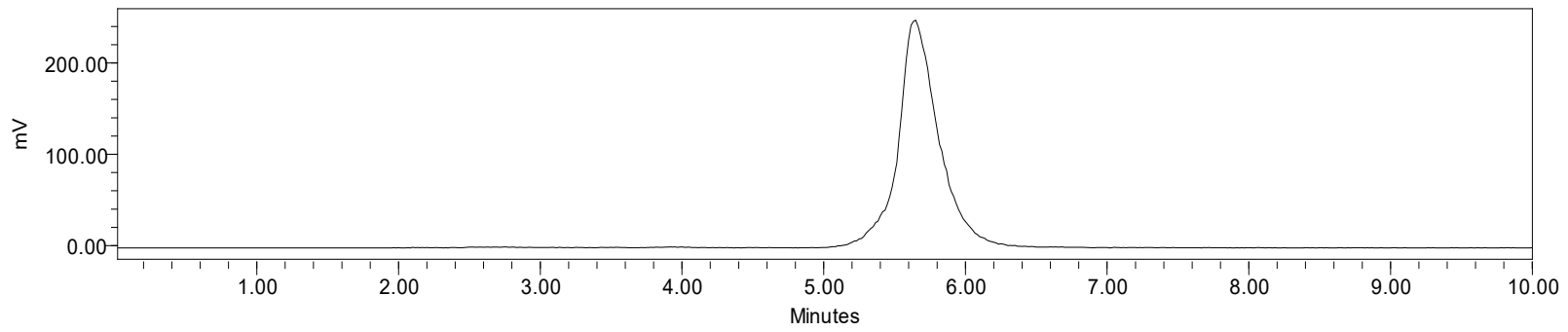

D. Analytical UV HPLC trace for $\left[{ }^{11} \mathrm{C}\right] \mathbf{1 2}, \mathrm{t}_{\mathrm{R}}=5.52 \mathrm{~min}$

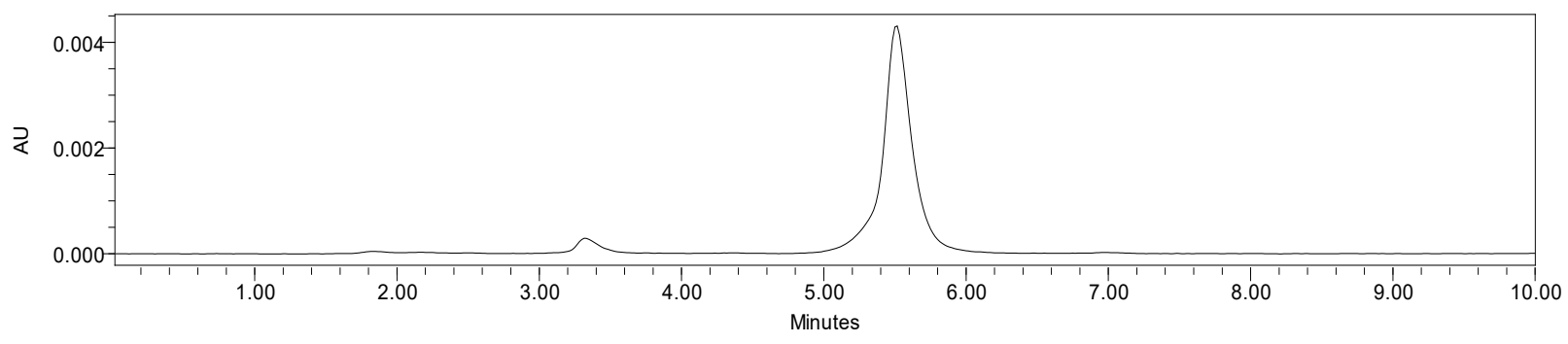




\section{Scheme 1.}
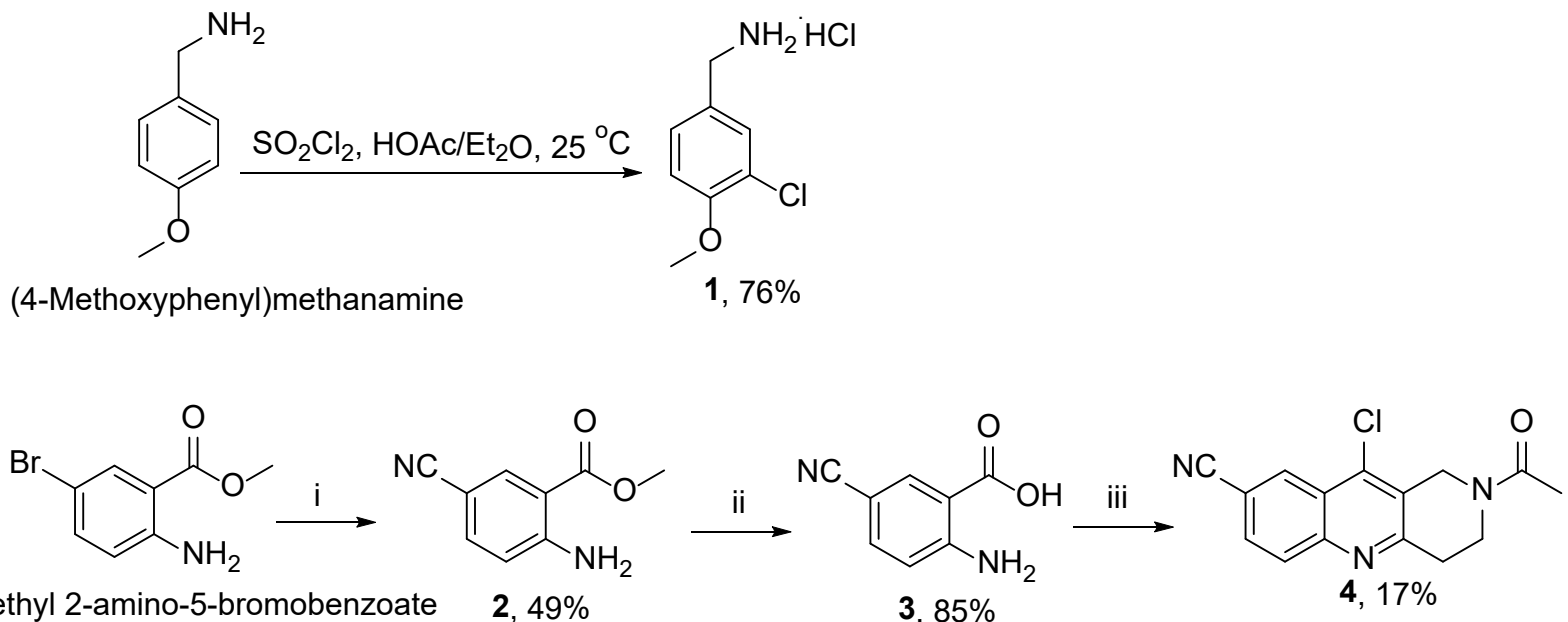

Methyl 2-amino-5-bromobenzoate $\quad 2,49 \%$

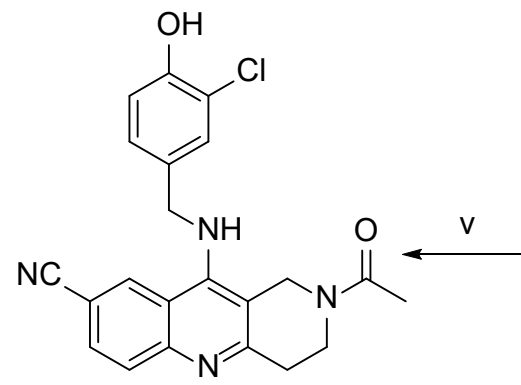

6, $30 \%$
$4,17 \%$

iv<smiles>COc1ccc(CNc2c3c(nc4ccc(C#N)cc24)CCN(C(C)=O)C3)cc1Cl</smiles> 


\section{Scheme 2.}

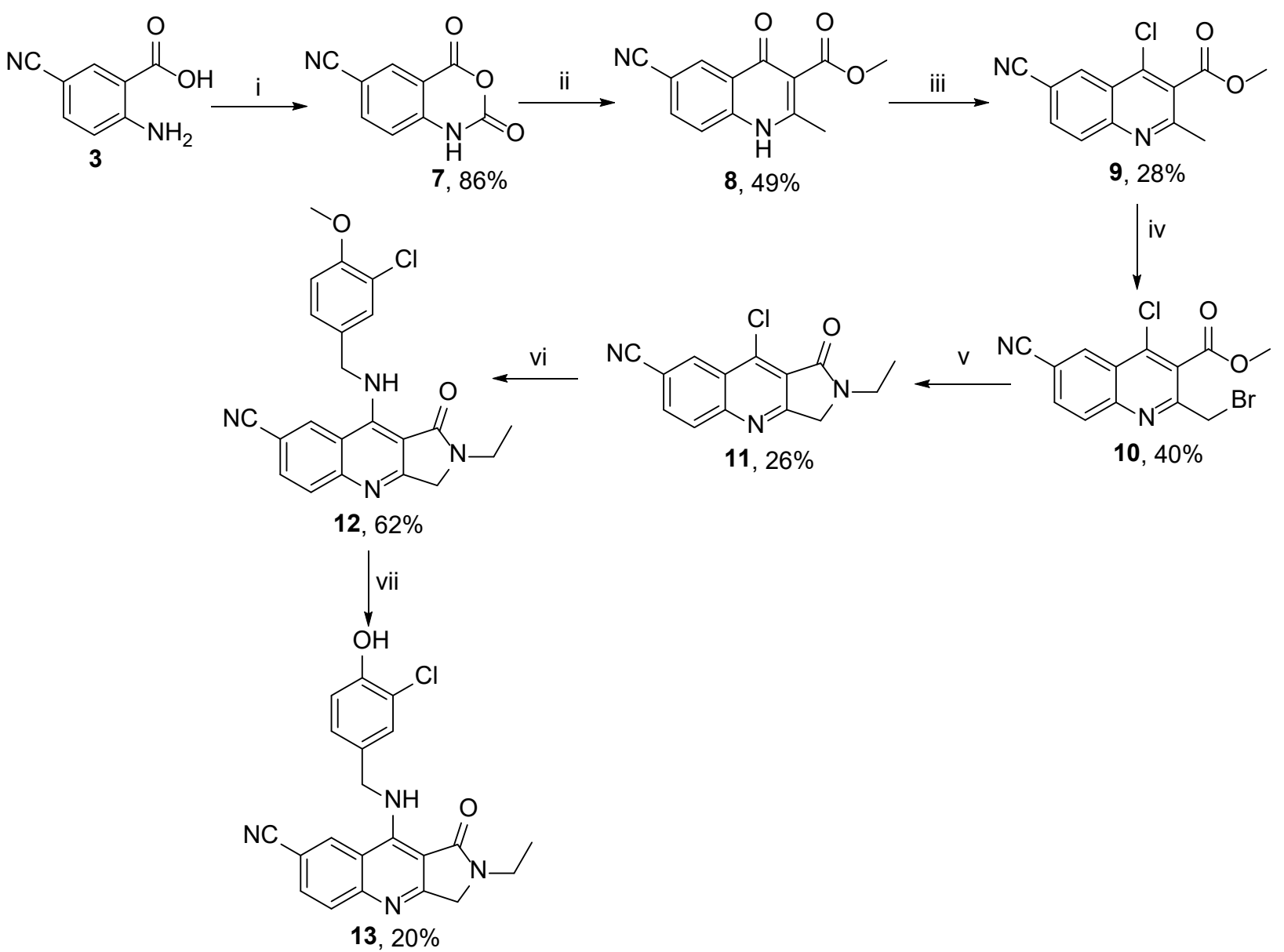


Scheme 3.
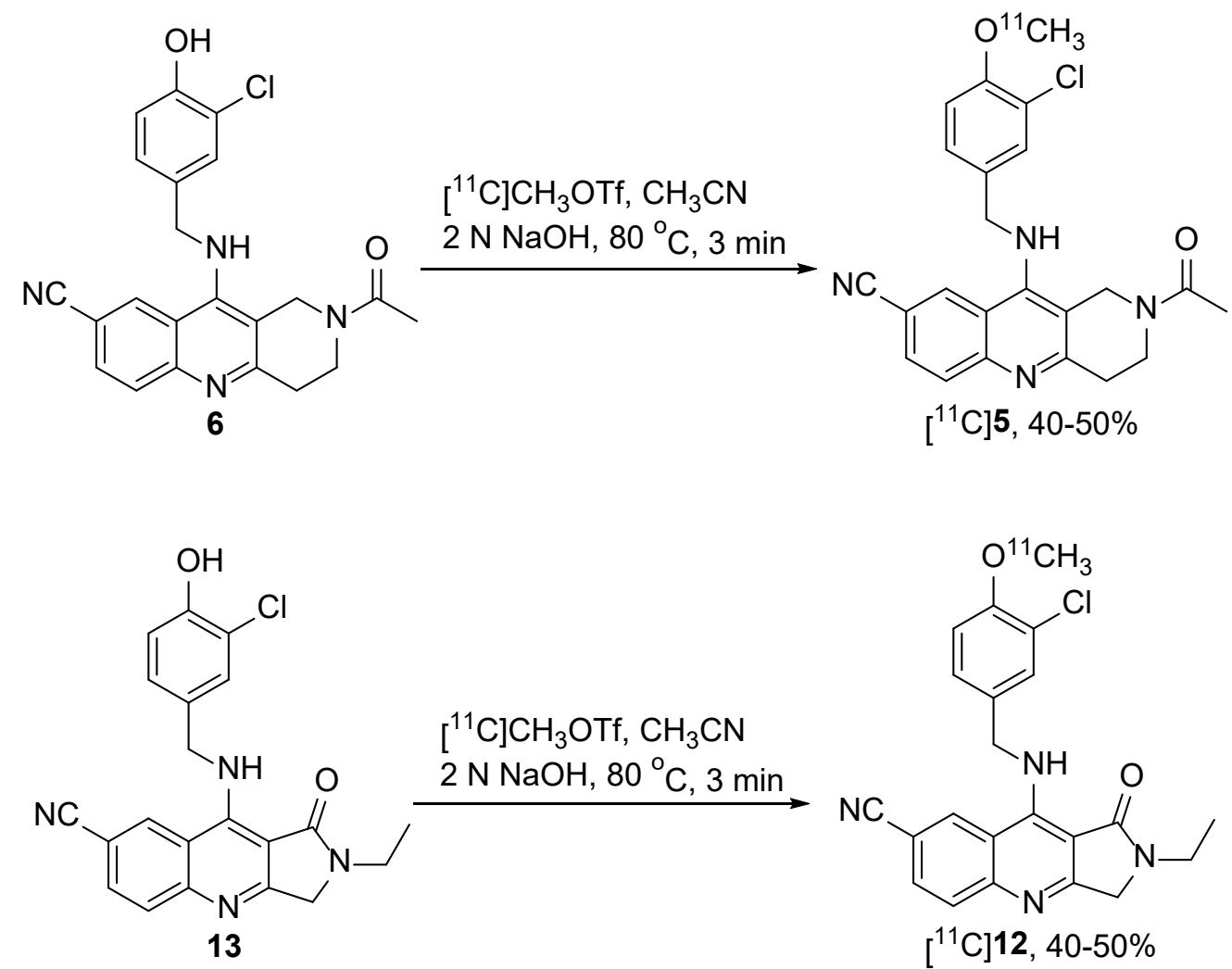